\title{
Kernel Bandwidth
}

National Cancer Institute

\section{Source}

National Cancer Institute. Kernel Bandwidth. NCI Thesaurus. Code C79423.

A scalar argument to a kernel function that determines what range of the nearby data points will be heavily weighted in making an estimate. 